\title{
Raised concentrations of aldehyde lipid peroxidation products in premature infants with chronic lung disease
}

Tohru Ogihara, Kazuya Hirano, Takao Morinobu, Han-Suk Kim, Mayo Hiroi, Hiromi Ogihara, Hiroshi Tamai

\begin{abstract}
Aim-To indicate the extent of lipid peroxidation induced by oxidative stress, by measuring aldehyde end products in biological samples.

Methods-A highly specific gas chromatography and mass spectrometry (GC/ MS) method was used to measure plasma concentrations of aliphatic aldehydes within the first week of life in 13 premature infants who subsequently developed chronic lung disease (CLD) and 11 infants without CLD (non-CLD). The oxime-tertbutyldimethylsilyl derivatives of aldehydes were analysed using 2,2,6,6- $\mathrm{d}_{4}-$ cyclohexanone as the internal standard.

Results-All of the aldehydes measured were raised in those infants with CLD compared with non-CLD infants. Plasma concentrations of heptanal, 2-nonenal, and 4-hydroxynonenal (HNE) were significantly increased in CLD infants on the day of birth, while the differences in all aldehydes between the two groups were not significant at 4-6 days of age. Logistic regression analysis showed that the increase in these three aldehydes within the first 24 hours of life independently showed significant associations with the development of CLD. In particular, an HNE concentration of $\geqslant 200 \mathrm{nM}$ on day 0 was the best predictor for the early detection of CLD (odds ratio $=32.0$ ), followed by a 2-nonenal concentration of $\geqslant 150 \mathrm{nM}$ $($ odds ratio $=16.0)$.
\end{abstract}

Conclusions-These findings suggest that lipid peroxidation may have a role in the pathogenesis of neonatal CLD.

(Arch Dis Child Fetal Neonatal Ed 1999;80:F21-F25)

Keywords: lipid peroxidation; aldehyde; 4-hydroxynonenal; gas chromatography and mass spectrometry

Department of Pediatrics, Division of Neonatology, Osaka Medical College, 2-7, Daigaku-machi, Takatsuki, Osaka, 569-0801, Japan

T Ogihara

K Hirano

T Morinobu

H-S Kim

M Hiroi

H Ogihara

H Tamai

Correspondence to: Dr Tohru Ogihara.

Accepted 18 August 1998
Although free radicals can injure many biological constituents, their effects on lipid molecules are most frequently used to evaluate free radical induced damage, often by measuring the end products of lipid peroxidation, such as aldehydes and hydrocarbon gases. ${ }^{6}$ Some noxious aldehydes, such as 4-hydroxynonenal (HNE), have also received attention as toxic secondary messengers, rather than simple inactive remnants of the lipid peroxidation process. $^{7}$ The thiobarbituric acid (TBA) test, which is supposed to detect malondialdehyde (MDA), is the one most frequently used to measure aldehydic end products. However, the TBA test has a low sensitivity and lacks specificity. ${ }^{6-8}$ To date, conflicting results have been obtained with respect to the concentrations of TBA reactive substances in biological samples from infants with CLD. ${ }^{9-11}$

In this study we attempted direct measurement of various aliphatic aldehyde concentrations in plasma using highly specific and sensitive gas chromatography and mass spectrometry (GC/MS), to overcome the disadvantages of the TBA test and to determine whether lipid peroxidation is involved in the development of CLD. We also measured the concentrations of $\alpha$-tocopherol and bilirubin as major antioxidants protecting against lipid peroxidation in plasma. As far as we are aware, this is the first report on the application of GC/MS for measuring aldehydes in newborn infants.

\section{Methods}

Inclusion criteria were: gestational age $<31$ weeks; mechanical ventilation at birth with a diagnosis of respiratory distress syndrome (RDS); survival > 40 days; absence of congenital heart disease, multiple malformations, documented chromosomal abnormality, congenital infection, and confirmed sepsis; and availability of two serial plasma samples obtained on days 0 and $4-6$. We obtained blood samples diluted with heparin by salvaging the remnants left after clinically indicated analysis of arterial blood gases was performed.

A total of 24 infants fulfilled the above criteria from among those admitted to the neonatal intensive care unit at Osaka Medical College between September 1995 and January 1998. They comprised 13 infants who had developed CLD (CLD group) and 11 infants with limited RDS (non-CLD group). The clinical characteristics of these 24 infants are summarised in table 1 . Fetal distress was defined by the pres- 
ence of cardiotocographic abnormalitiesbaseline heart rate $<100 / \mathrm{min}$ or late decelerations. RDS was diagnosed from a combination of the characteristic clinical features and radiographic criteria along with negative bacterial cultures, and was confirmed by a stable microbubble test of the gastric aspirate. ${ }^{12}$

Because of severe respiratory distress, 11 CLD infants and seven non-CLD infants received surfactant replacement with a single dose of surfactant TA (Tokyo Tanabe Co, Tokyo, Japan ) at a median time of 30 minutes after birth (range: $2-180 \mathrm{~min}$ ). About a third of the neonates were ventilated by intermittent mandatory ventilation (IMV) at a rate of 10-40 breaths/min, a peak pressure of $15-20 \mathrm{~cm} \mathrm{H}_{2} \mathrm{O}$, and a positive end expiratory pressure of 3-5 $\mathrm{cm} \mathrm{H}_{2} \mathrm{O}$. The other infants received high frequency oscillatory ventilation (HFO) at a frequency of 15 cycles/s and a mean airway pressure of $10-15 \mathrm{~cm} \mathrm{H}_{2} \mathrm{O}$ due to hypercapnoea $\left(\mathrm{PaCO}_{2}>60 \mathrm{mmHg}\right)$ or fighting uncoordinated IMV. A transcutaneous oxygen monitor was used to monitor arterial oxygen tension to ensure that it was maintained at 60-80 $\mathrm{mm} \mathrm{Hg}$. CLD was defined as the requirement for extra oxygen supplementation beyond 28 days of age associated with symptoms of persistent respiratory distress and hazy lung fields on chest $x$-ray films. ${ }^{13}$ No infant had a plasma IgM concentration $>20 \mathrm{mg} / \mathrm{dl}$ or a positive acute phase reactant score at birth.

Immediately after collection, blood was centrifuged at $3000 \times g$ for 10 minutes at $4^{\circ} \mathrm{C}$ to obtain plasma and stored at $-80^{\circ} \mathrm{C}$ under nitrogen. All infants received intravenous glucose during the study period and broad spectrum antibiotics for at least the first 3 days of life. No infant received a blood transfusion within the study period.

The study protocol was approved by the ethics committee of our college hospital and investigations were performed only after informed parental consent had been obtained.

Table 1 Clinical characteristics of study neonates

\begin{tabular}{lll}
\hline & $C L D(n=13)$ & non-CLD (n=11) \\
\hline Gestational age (weeks) & $27(24-29)$ & $28(26-30)$ \\
Birthweight (g) & $1065(694-1320)$ & $1168(626-1408)$ \\
Male & $9 / 13$ & $6 / 11$ \\
Fetal distress & $5 / 13$ & $3 / 11$ \\
Caesarean section & $10 / 13$ & $8 / 11$ \\
Apgar score & & \\
At 1 min & $5(1-8)$ & $6(1-8)$ \\
At 5 min & $7(2-10)$ & $9(1-10)$ \\
Surfactant therapy & $11 / 13$ & $7 / 11$ \\
HFO/IMV & $8 / 5$ & $7 / 4$ \\
Mean ventilator airway pressure (cm $\left.\mathrm{H}_{2} \mathrm{O}\right)$ & $13(8-15)$ & $13(10-15)$ \\
Total extra oxygen supplementation (\% hours) & & \\
$\quad$ Within 24 hours & $674(226-906)$ & $566(23-1616)$ \\
$\quad$ Within 7 days & $2071(371-5252)$ & $1257(68-5002)$ \\
Duration of intubation (days) & $10(4-83)^{\star}$ & $4(1-20)$ \\
Extubation by 7 days of life & $2 / 13$ & $4 / 11$ \\
Duration of oxygen supplementation (days) & $61(38-126)^{\star}$ & $10(1-64)$ \\
Duration of phototherapy within 7 days of life (hours) & $48(0-144)$ & $36(0-111)$ \\
Urine output (ml/kg/h) & & \\
$\quad$ Day 0 & $1.4(0.3-2.7)$ & $0.8(0.2-2.7)$ \\
$\quad$ Days 4-6 & $2.9(2.3-5.6)$ & $3.4(2.3-4.5)$ \\
Age at the beginning of enteral feeding (days) & $8(4-25)$ & $6(3-10)$ \\
Start of enteral feeding by 7 days of life & $5 / 13$ & $5 / 11$
\end{tabular}

Values are expressed as median and range. Differences between groups are assessed by Mann-Whitney U-test or Fisher's exact test as appropriate.

${ }^{\star} \mathrm{p}<0.05$ vs non-CLD infants.
SAMPLE PREPARATION FOR GC/MS ASSAY

For oxime-tert-butyldimethylsilyl derivatisation of plasma aldehydes, we applied the method used for isolated rat hepatocytes. ${ }^{14}$ Deuterated cyclohexanone $\quad\left(2,2,6,6-\mathrm{d}_{4}\right.$-cyclohexanone; Isotec Inc. Miamisburg, $\mathrm{OH}$, USA) was used as the internal standard instead of $\left[1-{ }^{14} \mathrm{C}\right] \mathrm{cy}-$ clohexanone as in the original method. d4cyclohexanone ( $25 \mathrm{pmol}$ ) was added to $50 \mu \mathrm{l}$ of plasma and the mixture was reacted with $1 \mathrm{ml}$ of $0.6 \mathrm{M} \mathrm{HClO}$ containing $100 \mathrm{mg}$ of hydroxylamine hydrochloride at room temperature in the dark for 15 minutes. Subsequent procedures were the same as in the original method.

ANALYSIS OF ALDEHYDES

A Shimadzu QP-5000 version 2 GC/MS system (Shimadzu Corp., Kyoto, Japan) equipped with a DB-1 capillary column ( $30 \mathrm{~m}$ $\times 0.25 \mathrm{~mm}, 0.25 \mathrm{~mm}$ film thickness; J \& W Scientific, Folsom, CA, USA) was used for aldehyde analysis. The operating conditions were the same as reported before. ${ }^{14}$

The plasma $\alpha$-tocopherol concentration was measured using high performance liquid chromatography (HPLC) with electrochemical detection, according to the method used in our previous report. ${ }^{15}$ Plasma total bilirubin was determined using the alkaline diazo-coupling method. ${ }^{16}$

The total extra oxygen supplementation for each subject was determined using the following equation:

$\sum$ total extra oxygen supplementation = administered oxygen concentration [\%] (fraction of inspired oxygen-21) $\times$ duration [hours]. ${ }^{3}$

Comparison of characteristic variables between groups was performed using the MannWhitney $U$ test for continuous variables and Fisher's exact test for categorical data. Intergroup differences in plasma aldehyde concentrations were determined using the MannWhitney U test with Bonferroni's adjustment for comparison of multiple time points. Logistic regression analysis was used to evaluate whether plasma aldehyde concentrations on the day of birth were associated with the development of CLD. The estimated $\beta$ coefficients and odds ratios were given. We also calculated the odds ratios for the relative risk of CLD at a plasma heptanal concentration $\geqslant 500 \mathrm{nM}$, a 2 -nonenal concentration $\geqslant 150 \mathrm{nM}$, and an HNE concentration $\geqslant 200 \mathrm{nM}$, within the first 24 hours of life using Fisher's exact test; $\mathrm{p}<$ 0.05 was considered significant. Data were analysed using Statview 4.0 (Abacus Concepts Inc., Berkeley, CA) or Statistical Analysis System 6.11 (SAS Institute, Inc., Cary, NC) statistical software packages.

\section{Results}

There were no significant differences between the infants with and without CLD in gestational age, birthweight, gender, the presence of fetal distress, the delivery method, and the Apgar scores at 1 and 5 mins (table 1). There were also no differences between the two groups in incidence of surfactant administra- 
Table 2. Plasma concentrations of alehydes (nM), $\alpha$-tocopherol $(\mu M)$, and bilirubin (mM) in CLD and non-CLD infants within first week of life

\begin{tabular}{llllll}
\hline & \multicolumn{2}{l}{ Day 0} & & & \multicolumn{2}{l}{ Days 4-6 } & \\
\cline { 2 - 3 } \cline { 5 - 6 } \cline { 5 - 6 } & $C L D$ & non-CLD & & & \multicolumn{2}{l}{ CLD } & non-CLD \\
\hline Sampling time (hours) & $7(2-19)$ & $4(2-18)$ & & $144(96-168)$ & $116(96-144)$ \\
Pentanal (nM) & $268(138)$ & $212(115)$ & & $286(191)$ & $272(128)$ \\
Hexanal & $1970(741)$ & $1605(779)$ & & $1634(995)$ & $1564(962)$ \\
2-Hexanal & $70(28)$ & $52(18)$ & & $269(398)$ & $96(44)$ \\
Heptanal & $1013(1033)^{\star}$ & $342(330)$ & & $981(990)$ & $494(462)$ \\
2-Heptanal & $120(74)$ & $95(81)$ & & $116(84)$ & $96(99)$ \\
2-Octenal & $77(46)$ & $53(39)$ & & $110(48)$ & $82(54)$ \\
2-Nonenal & $190(163)^{\star}$ & $70(50)$ & & $111(78)$ & $98(67)$ \\
4-Hydroxy-nonenal & $250(60)^{\star}$ & $137(95)$ & & $211(55)$ & $159(131)$ \\
$\alpha$-tocopherol $(\mu M)$ & $5.0(1.9)$ & $5.0(2.8)$ & & $6.3(1.9)$ & $5.6(2.3)$ \\
Bilirubin $(m M)$ & $8.4(2.4)$ & $8.4(3.6)$ & & $12.4(2.5)$ & $14.7(3.9)$ \\
\hline
\end{tabular}

Values are expressed as the mean (SD) or the median (range). Differences between groups are assessed by Mann-Whitney U-test.

$\star \mathrm{p}<0.025$ vs non-CLD infants.

Table 3 Logistic regression analysis of independent association of three significantly increased plasma aldehydes within first 24 hours of life with development of CLD

\begin{tabular}{llllll}
\hline Aldehyde $(n M)$ & $\beta$ & $S E$ & $p$ & $O R$ & $95 \% C I$ \\
\hline Heptanal & 0.00266 & 0.00135 & 0.0495 & 1.003 & $1.00001-1.00532$ \\
2-Nonenal & 0.0171 & 0.00823 & 0.0375 & 1.017 & $1.00097-1.03379$ \\
4-Hydroxy-nonenal & 0.0199 & 0.00825 & 0.0161 & 1.020 & $1.00374-1.03673$
\end{tabular}

$\mathrm{SE}=$ standard error; $\mathrm{OR}=$ odds ratio $\mathrm{CI}=$ confidence intervals .

Table 4 Development of CLD and concentrations of plasma aldehyde within first 24 hours of life

\begin{tabular}{llll}
\hline Aldehyde $(n M)$ & $O R$ & $p$ & $95 \% C I$ \\
\hline Heptanal $\geqslant 500 \mathrm{nM}$ & 6.0 & 0.0995 & $1.041-34.573$ \\
2-Nonenal $\geqslant 150 \mathrm{nM}$ & 16.0 & 0.0131 & $1.961-130.548$ \\
4 -Hydroxy-nonenal $\geqslant 200 \mathrm{nM}$ & 32.0 & 0.0022 & $3.856-265.553$
\end{tabular}

$\mathrm{OR}=$ odds ratio; $\mathrm{CI}=$ confidence intervals.

tion, modality of ventilation, mean ventilator airway pressure, and total extra oxygen supplementation. Although the duration of intubation and oxygen supplementation was longer in the CLD group, the numbers of infants extubated during the study period did not differ significantly between the groups. Enteral feeding was started in five CLD and five non-CLD infants by 7 days of life; the difference was not significant.

Table 2 shows that the order of predominance for aldehydes was hexanal, heptanal, pentanal, HNE. All of the measured aldehydes were raised at both sampling times in the CLD infants compared with the non-CLD infants. The plasma concentrations of heptanal, 2-nonenal, and HNE were significantly increased in CLD infants on the day of birth, but the differences between the two groups did not reach significance at 4-6 days of age. The $\alpha$-tocopherol and bilirubin concentrations did not differ between the two groups throughout the study.

Table 3 shows the results of logistic regression analysis to estimate the independent association of the three significantly increased aldehydes on the day of birth with the development of CLD. Each aldehyde was significantly associated with CLD, with HNE topping the list.

The typical distribution of each aldehyde on day 0 is shown in fig 1 . From the distribution patterns, we calculated the odds ratio (OR) for each aldehyde using cutoff values of $500 \mathrm{nM}$ for heptanal, $150 \mathrm{nM}$ for 2-nonenal, and 200 $\mathrm{nM}$ for $\mathrm{HNE}$ in association with the relative risk for the development of CLD (table 4). An $\mathrm{HNE}$ concentration $\geqslant 200 \mathrm{nM}$ within 24 hours after birth was the best predictor for the early detection of CLD (OR=32.0, p<0.01), followed by 2 -nonenal $\geqslant 150 \mathrm{nM} \quad(\mathrm{OR}=16.0$, $\mathrm{p}<0.02$ ).

\section{Discussion}

Based on the quantitative profiles of aldehydes reportedly formed during autoxidation of fatty acids, such as oleic, linoleic, $\alpha$-linolenic, $\gamma$-linolenic, and arachidonic acid, ${ }^{17}{ }^{18}$ we measured eight aldehydes in this study (pentanal, hexanal, 2-hexenal, heptanal, 2-heptenal, 2-octenal, 2-nonenal, and HNE). Pentanal and hexanal are mainly generated from the peroxidation of linoleic and arachidonic acid, while heptanal is generated from oleic acid, and 2-hexenal from $\alpha$-linolenic acid. 2-nonenal and HNE are also the main peroxidation products of $\gamma$-linolenic and arachidonic acid. ${ }^{17}$ Among these aldehydes, HNE has already been widely studied ${ }^{19}$ and hexanal has also been reported ${ }^{20}$ to be an indicator of lipid peroxidation. It has already been confirmed that not only hexanal, HNE, and MDA, but also other aldehydes, actually increase during peroxidation of rat liver microsomes and in rats loaded with ethanol. ${ }^{14} 17$

All of the aldehydes measured in this study were higher in the CLD infants than in the non-CLD infants during the first week of life. The plasma concentrations of heptanal, 2-nonenal, and HNE were significantly increased within 24 hours of birth when compared with those in non-CLD infants. This finding clearly indicates that CLD infants were
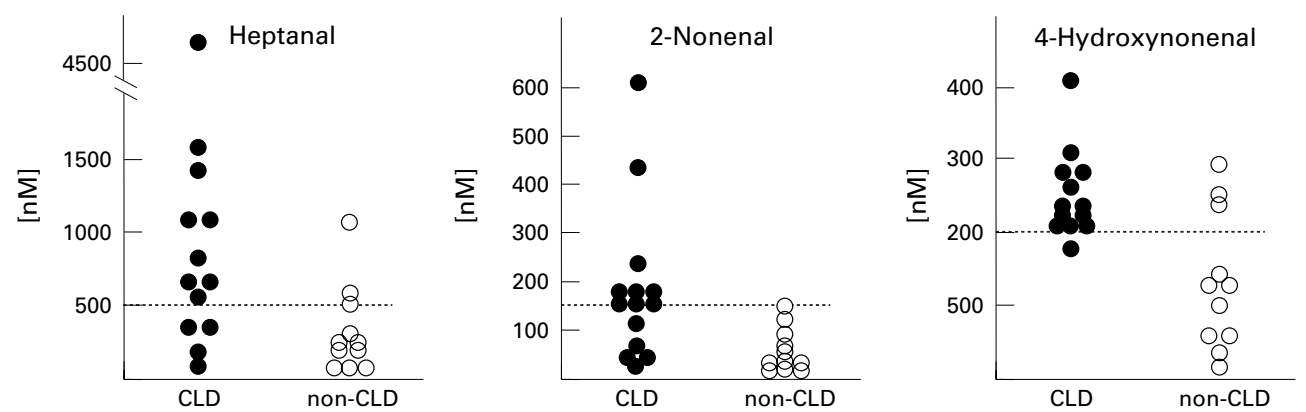

Figure 1 Concentrations (nM) of heptanal (left), 2-nonenal (middle), and 4-hydroxynonenal (right) in plasma of the infants with or without CLD within the first 24 hours of life. Closed circles represent values in CLD infants and open circles those in non-CLD infants. Dotted lines indicate cutoff levels to estimate relative risk for the development of CLD. 
exposed to more severe lipid peroxidation than non-CLD infants on the day of birth. Aldehyde concentrations in umbilical cord plasma are known to be increased in infants with perinatal hypoxia compared with those without it. ${ }^{21}$ In this study, however, fetal distress and the Apgar scores were nearly identical in both groups. Thus the difference in aldehyde concentrations between the two groups was not attributable to perinatal hypoxia and reperfusion injury.

Excessive lipid peroxidation may be caused by an imbalance between free radical generation and antioxidant defence capacities. However, if excessive free radical formation occurred in CLD infants, this did not seem to be due to hyperoxia itself, because the ventilation method and total extra oxygen supplementation were comparable in the two groups. As rapid influx of neutrophils into the lungs is observed just after delivery in infants with RDS, ${ }^{22}$ activated neutrophils may be a primary source of lipid peroxidation inducing free radicals. Although $\alpha$-tocopherol and bilirubin concentrations were almost equal in the two groups, we cannot exclude a decrease in total antioxidant capacity in CLD infants on day 0 , because various low molecular weight antioxidants in plasma seem to work synergistically, providing greater protection against free radical damage than could be achieved by any single antioxidant acting alone. ${ }^{23}$ Furthermore, we did not investigate the status of important antioxidants such as enzymatic antioxidants or chelators of transitional metal ions.

The differences in aldehyde concentrations at 4-6 days of age did not reach significance. Although the number of infants who received enteral feeding within the study period was the same in each group and all were only given breast milk, the effect of feeding cannot be ignored, because aldehyde concentrations could be influenced by the plasma lipid content. We recommend that future studies consider lipid intake and the timing of blood sampling.

Our most interesting finding was that heptanal, 2-nonenal, and HNE concentrations on day 0 might predict the development of CLD. In particular, an HNE concentration $\geqslant$ $200 \mathrm{nM}$ and a 2-nonenal concentration of $\geqslant$ $150 \mathrm{nM}$ on the day of birth are excellent indicators for the early detection of CLD. As HNE is known to be highly cytotoxic, ${ }^{7}$ it may also have a role as a toxic secondary messenger in the lipid peroxidation process.

Application of the common TBA test to body fluids and tissue extracts poses several problems, ${ }^{6-8}$ the most serious of which is that many chemicals other than MDA act as false positive chromogens. Investigations using the usual TBA test have failed to demonstrate increased concentrations of TBA reactive substances in the serum ${ }^{9}$ or lung tissue ${ }^{10}$ of CLD infants compared with non-CLD infants.

In contrast to the TBA test, GC/MS analysis of aldehydes is highly specific and sensitive, ${ }^{6}$ and is believed to be one of the most precise methods currently available. A fundamental difficulty of measuring aldehydes is that most commercial HPLC grade organic solvents con- tain aldehyde contaminants. ${ }^{24}$ Therefore, we offset aldehyde contaminants in the solvent by running a blank every 10 samples. Furthermore, the method used in this study could not be applied to the measurement of MDA, which is reported to be another major aldehyde formed during lipid peroxidation. ${ }^{17}$ Using a specific HPLC method, the serum concentration of MDA-TBA complexes was significantly higher in CLD infants than in non-CLD infants at 7 days of age. ${ }^{11}$ In the case of hydrocarbon gases, another highly specific marker, a close correlation between free radical induced lipid peroxidation and outcome for very low birthweight infants with RDS has been demonstrated by quantitating ethane and pentane in expired air during the first five days of life. ${ }^{25}$ These reports are consistent with our present findings.

To our knowledge, no previous studies have assessed the same aldehydes in newborn infants, except for HNE. Recently, Schmidt et al determined the HNE concentration in umbilical cord serum from neonates using HPLC. ${ }^{21}$ According to their report, HNE concentrations increased with gestational age and the HNE concentration at 33 weeks of gestation was about $100 \mathrm{nM}$. This value is comparable with our data on HNE concentrations on the day of birth in both groups, despite the difference in analytical methods. Furthermore, the order of predominance and the approximate range of plasma aldehyde concentrations were almost identical with those recently reported by Yazdanpanah et al, ${ }^{26}$ who measured 18 aldehydes by GC/MS in children with various cancers.

The most prominent pathological change occurring in CLD infants is the overproduction of connective tissue. There has been increasing clinical evidence of the involvement of oxidative stress and lipid peroxidation in various fibrotic conditions affecting the liver, lungs, arteries, and nervous system. A growing body of data show that lipid peroxidation and aldehydic end products, such as HNE, induce genetic over expression of fibrogenic cytokines, especially transforming growth factor $\beta$, as well as increased transcription and synthesis of collagen. ${ }^{27}$ Kotecha et al reported increased concentrations of transforming growth factor $\beta-1$ in the bronchoalveolar lavage fluid of CLD infants. ${ }^{28}$ Therefore, aldehydes such as HNE may be a possible fibrosis inducing factor, and the significant increased concentrations of some aldehydes in CLD infants may be an important direct link to the development of this condition beyond the mere fingerprint of lipid peroxidation. Further studies are needed to clarify the precise effect of lipid peroxidation on fibrotic changes in the lungs of CLD infants.

We conclude that significantly increased plasma concentrations of some aldehydes in CLD infants on the day of birth strongly suggest the involvement of free radicals in the development of CLD. Furthermore, plasma $\mathrm{HNE}$ and 2-nonenal concentrations within 24 hours of birth seem to be excellent predictors for the early detection of CLD. These data may 
help to improve our understanding of the role of free radicals in the progression of CLD.

We thank Drs M Mino, A Nagai, H Moji, H Tanaka, T Ogawa, We thank Drs M Mino, A Nagai, H Moji, H Tanaka, T Ogawa, M Imanishi, M Kobayashi, and H Ikeuchi for excellent labora$M$ Imanishi, M Kobayashi, and H Ikeuchi for excellent labora-
tory assistance. We are also grateful to T Nakanishi, E Hata, and tory assistance. We are also grateful to $\mathrm{T}$ Nakanishi, E Hata, and the nursing staff

ple collection.
This work was supported by a Grant-in-Aids ( 00211128 ) for Scientific Research from the Ministry of Education, Science, and Culture of Japan, and a Grant for Pediatric Research (8C-2) from the Ministry of Health and Welfare.

This article is dedicated to the memory of Makiko Arakawa.

1 Northway Jr WH. Bronchopulmonary dysplasia: then and now. Arch Dis Child 1990;65:1076-81.

2 Saugstad OD. Oxygen toxicity in the neonatal period. Acta Paediatr Scand 1990;79:881-92.

3 Ogihara T, Okamoto R, Kim HS, et al. New evidence for the involvement of oxygen radicals in triggering neonatal chronic lung disease. Pediatr Res 1996;39:117-9.

4 Ogihara T, Kim HS, Hirano K, et al. Oxidation products of uric acid and ascorbic acid in preterm infants with chronic lung disease. Biol Neonate 1998;73:24-33.

5 Moison RMW, Beaufort AJ, Haasnoot AA, Dubbelman TMAR, Zoeren-Grobben D, Berger HM. Uric acid and ascorbic acid redox ratios in plasma and tracheal aspirate of preterm babies with acute and chronic lung disease. Free of preterm babies with acute and chro
Radical Biol Med 1997;23:226-34

6 Halliwell B, Chirico S. Lipid peroxidation : its mechanism, measurement, and significance. Am 7 Clin Nutr measurement, and significa

7 Esterbauer H, Schaur RJ, Zollner H. Chemistry and biochemistry of 4-hydroxynonenal, malonaldehyde and related aldehydes. Free Radical Biol Med 1991;11:81-128

8 Gutteridge JMC. Aspects to consider when detecting and measuring lipid peroxidation. Free Radical Res Commun 1986;1:173-84.

9 McCarthy K, Bhogal M, Nardi M, Hart D. Pathogenic factors in bronchopulmonary dysplasia. Pediatr Res 1984;18:483-7.

10 Strange RC, Cotton W, Fryer AA, Jones P, Bell J, Hume R. Lipid peroxidation and expression of copper-zinc and manganese superoxide dismutase in lungs of premature infants with hyaline membrane disease and bronchopulinfants with hyaline membrane disease and bronch

11 Inder TE, Graham P, Sanderson K, Taylor BJ. Lipid peroxidation as a measure of oxygen free radical damage in the
very low birthweight infant. Arch Dis Child 1994;70:F107F11.

12 Pattle RE, Kratzing CC, Parkinson CE, et al. Maturity of fetal lungs tested by production of stable microbubbles in amniotic fluid. Br f Obstet Gynecol 1979;86:615-22.
13 Ogawa Y, Fujimura M, Goto A, et al. Epidemiology of neonatal chronic lung disease in Japan. Acta Paediatr fpn 1992;34:663-7.

14 Norsten-Höög C, Cronholm T. Analysis of aldehydic lipid peroxidation products in rat liver and hepatocytes by gas chromatography and mass spectrometry of the oxime-tertbutyl-dimethylsilyl derivatives. Anal Biochem 1990;189:131-7.

15 Tamai H, Manago M, Yokota K, Kitagawa M, Mino M. Determination of $\alpha$-tocopherol in buccal mucosal cells using electrochemical detector. Internat f Vit Nutr Res 1988;58:202-7.

16 Michaëlsson M, Nosslin B, Sjölin S. Plasma bilirubin determination in the newborn infant - A methodological study with special reference to the influence of hemolysis. Pediatrics 1965;35:925-31.

17 Esterbauer $\mathrm{H}$, Zollner H, Schaur RJ. Aldehydes formed by lipid peroxidation : mechanisms of formation, occurrence, and determination. In: Vigo-Pelfrey C, ed. Membrane Lipid Oxidation. Boca Raton, FL: CRC Press, 1990:239-68.

18 Grosch W. Reactions of hydroperoxides - products of low molecular weight. In: Chan HWS, ed. Autoxidation of unsaturated lipids. London: Academic Press, 1987:95-137.

19 Blasig IE, Grune T, Schönheit K, Rohde E, Jakstadt M, Haseloff RF, Siems WG. 4-Hydroxynonenal, a novel indicator of lipid peroxidation for reperfusion injury of the myocardium. Am f Physiol 1995;269(1 Pt 2):H14-H22.

20 Frankel EN, Hu ML, Tappel AL. Rapid headspace gas chromatography of hexanal as a measure of lipid peroxidation in biological samples. Lipids 1989;24:976-81.

21 Schmidt H, Grune T, Müller R, Siems WG, Wauer RR. Increased levels of lipid peroxidation products malondialdehyde and 4-hydroxy-nonenal after perinatal hypoxia. Pediatr Res 1996;40:15-20.

22 Ogden BE, Murphy SA, Saunders GC, Pathak D, Johnson JD. Neonatal lung neutrophils and elastase / proteinase inhibitor imbalance. Am Rev Respir Dis 1984;130:817-21.

23 Niki E. Antioxidants in relation to lipid peroxidation.Chem Phys Lipid 1987; 44:227-53.

24 Holley AE, Walker MK, Cheeseman KH, Slater TF. Measurement of n-alkanals and hydroxyalkenals in biological samples. Free Radical Biol Med 1993;15:281-9.

25 Pitkänen OM, Hallman M, Andersson SM. Correlation of free oxygen radical-induced lipid peroxidation with outcome in very low birth weight infants. $f$ Pediatr 1990;116:760-4.

26 Yazdanpanah M, Luo X, Lau R, Greenberg M, Fisher LJ, Lehotay DC. Cytotoxic aldehydes as possible markers for childhood cancer. Free Radical Biol Med 1997;23:870-8.

27 Poli G, Parola M. Oxidative damage and fibrogenesis. Frer Radical Biol Med 1997;22:287-305.

28 Kotecha S, Wangoo A, Silverman M, Shaw RJ. Increase in the concentration of transforming growth factor beta- 1 in bronchoalveolar lavage fluid before development of chronic lung disease of prematurity. $\mathcal{F}$ Pediatr 1996;128:464-9. 